\title{
Regulation of Adult Neurogenesis by Antidepressant Treatment
}

\author{
Ronald S. Duman, Ph.D., Shin Nakagawa, M.D., and Jessica Malberg, Ph.D.
}

Demonstration of neurogenesis in adult brain represents a major advance in our understanding of the cellular mechanisms underlying neuronal remodeling and complex behavior. Recent studies from our laboratory and others demonstrate that chronic administration of an antidepressant, including either a 5-HT or norepinephrine selective reuptake inhibitor, up-regulates neurogenesis in adult rodent hippocampus. Up-regulation of neurogenesis could block or reverse the effects of stress on hippocampal neurons, which include down-regulation of neurogenesis, as well as atrophy. The possibility that the cAMP signal transduction cascade contributes to the regulation of neurogenesis by antidepressants is supported by previous studies and by recent work. Although additional studies must be conducted to determine the significance of adult neurogenesis in humans, these findings will stimulate new avenues of research to identify the cellular and molecular basis of stress-related mood disorders as well as the development of novel therapeutic strategies.

[Neuropsychopharmacology 25:836-844, 2001] (C) 2001 American College of Neuropsychopharmacology. Published by Elsevier Science Inc.
KEY WORDS: Stress; Depression; Fluoxetine; cAmp; CREB, Neurotrophic factor

Our understanding of how the brain functions and undergoes adaptation and plasticity has changed dramatically over the past several years. A great deal of work has demonstrated that altered levels of neurotransmitters, second messenger pathways, and gene expression profiles underlie cellular and behavioral plasticity. This includes models of learning and memory as well as models of depression, anxiety, and psychosis, and the

From the Division of Molecular Psychiatry, Abraham Ribicoff Research Facilities, Department of Psychiatry, Yale University School of Medicine, Connecticut Mental Health Center, 34 Park Street, New Haven, CT 06508, USA

Address correspondence to: Ronald S. Duman, Ph.D., Abraham Ribicoff Research Facilities, Yale University School of Medicine, 34 Park Street, New Haven, CT 06508, Tel: (203) 974-7726, Fax: (203) 974-7724, Email: ronald.duman@yale.edu

Received July 20, 2001; revised August 8, 2001; accepted August 9, 2001. long-term actions of psychotropic drugs. However, it is now becoming increasing evident that changes in cellular morphology and even more pronounced alterations in brain structure also contribute to neural plasticity or remodeling. At the cellular level these changes can occur in the form of up or down-regulation of synapse formation and spine density or extension and retraction of dendrites. Another very dramatic example is up or down-regulation of neurogenesis in adult brain. Studies in recent years demonstrate that new cell birth occurs in adult brain and that the rate of neurogenesis and the survival of new neurons is regulated by a number of environmental, endocrine, and pharmacological treatments.

In this paper we discuss the evidence that structural remodeling may be involved in the pathophysiology and treatment of mood disorders. The potential role of neurogenesis is also discussed as well as the molecular mechanisms which underlie antidepressant regulation of new cell birth and survival. The studies to date suggest an exciting possibility for the role of neurogenesis 
in depression, although this hypothesis must be viewed with caution until additional evidence is provided, particularly in humans.

\section{STRUCTURAL ALTERATIONS IN MOOD DISORDERS}

The possibility that cellular remodeling may contribute to the pathophysiology of mood disorders has gained support from clinical brain imaging studies. Much of this work has focused on the hippocampus, a limbic brain structure that plays a role in learning and memory as well as control of the hypothalamic-pituitary-adrenal (HPA) axis. There are now several independent studies reporting that the volume of hippocampus is decreased in patients suffering from depression or post traumatic stress disorder (PTSD) (Bremner et al. 1995, 2000; Sheline et al. 1996, 1999; Steffens et al. 2000). Sheline and colleagues have reported that the reduction in hippocampal volume correlates with the length of illness, suggesting that the stress associated with depression may cause the structural change and not the reverse (Sheline et al. 1999). However, it is possible that small structural changes may occur that contribute to the onset of depression and that the magnitude of the hippocampal reduction then continues to progress over the course of illness. It will be important to determine if the reduction in hippocampal volume is reversible with successful antidepressant treatment, and studies are currently underway at several sites to answer this question.

\section{REGULATION OF ADULT NEUROGENESIS}

The emphasis on studies of hippocampus stems in part from the work of McEwen and colleagues demonstrating that stress can have profound effects on the morphology of hippocampal neurons (see McEwen 1999; Gould et al. 1998). This includes a decrease in the dendritic arborization of CA3 pyramidal neurons and down-regulation of neurogenesis of granule cells in adult dentate gyrus. The focus of this paper will be on studies of neurogenesis. However, it is important to point out the potential significance of the CA3 neuronal atrophy because it is not yet clear if one or both of these stress effects is more likely to explain the reduction in hippocampal volume observed in depression and PTSD.

\section{Neurogenesis in Adult Brain}

Neurogenesis has been documented in adult brain of a number of different animals, including bird, rodent, monkey and human (see Gould et al. 1999; Gage 2000; Gross 2000). In adult mammalian brain, significant rates of adult neurogenesis are restricted to two brain regions, the olfactory bulb and the hippocampus. In the hippocampus, progenitor cells are located in the subgranular zone where they divide and give rise to new neurons. A recent detailed analysis of neurogenesis reports that there are approximately 9,000 new cells per day or 250,000 cells per month in adult rodent hippocampus (Cameron and McKay 2001). Approximately $50 \%$ of these cells differentiate and express cellular markers characteristic of neurons, which represents about $6 \%$ of the total number of granule cells. It is also estimated that this number of new granule neurons represents about $60 \%$ of the afferents from the entorhinal cortex and $30 \%$ of CA3 pyramidal cells receiving efferent projections from granule cells (Cameron and McKay 2001). These findings indicate that the number of new neurons, presumably with distinct characteristics, would be sufficient to contribute in a significant manner to the function of hippocampus. Exact determinations of the number of new neurons in primate brain have not been conducted, but estimates are that there may be 10 to $20 \%$ of the number of newborn cells observed in rodent brain. This amount of new neurons would be sufficient to influence the function of hippocampus in primates.

The possibility that neurogenesis plays a role in neural plasticity and remodeling is supported by reports

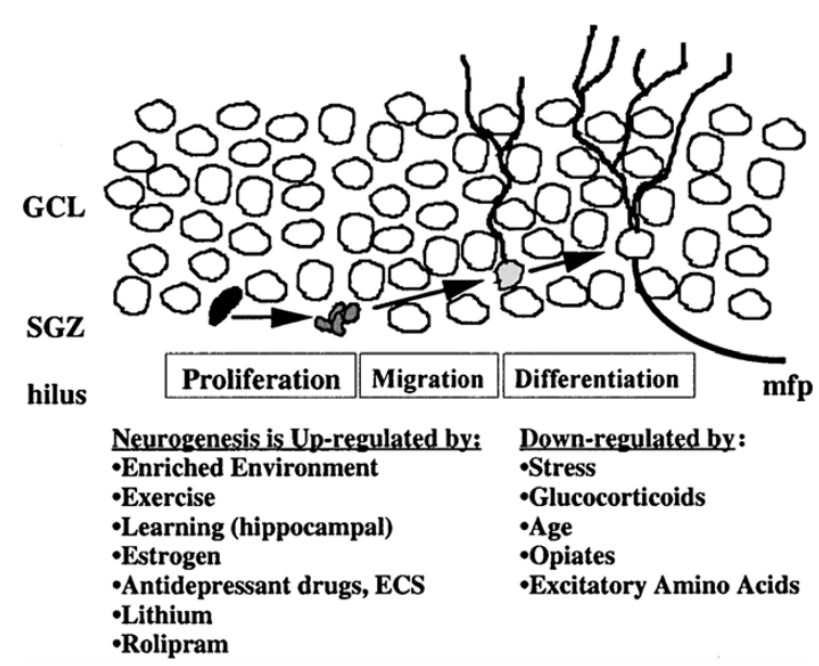

Figure 1. Model of adult neurogenesis in the granule cell layer of hippocampus. Neurogenesis is localized to the subgranular zone (SGZ) of the hippocampus, which is between the granule cell layer (GCL) and the hilus. Neural progenitor cells proliferate and give rise to new cells that differentiate into neurons and migrate into the granule cell layer. These new granule cells extend axons and dendrites and appear to integrate functionally into the existing granule cell layer. "mfp" identifies the mossy fiber pathway which projects to the CA3 pyramidal cell layer. Adult neurogenesis can be up or down-regulated by a number of stimuli, suggesting that neural adaptations in response to these treatments may involve regulation of neurogenesis. 
that neurogenesis in adult brain can be regulated by a number of treatments. Stimuli that increase neurogenesis include exercise, enriched environment, hippocampal-dependent learning, and estrogen (Figure 1) (see Tanapat et al. 1999; van Praag et al. 2000; Gould et al. 1999; Gage 2000). Alterations in neurogenesis can occur via regulation of the rate of cell proliferation or regulation of the number of newborn neurons that survive. Approximately $50 \%$ of the new neurons in adult rodent hippocampus survive while the remaining cells degenerate within four weeks after birth. More definitive evidence for a functional role of adult neurogenesis has been reported in a recent study. In this paper Shors and Gould demonstrate that inhibition of neurogenesis by administration of a chemical cell cycle inhibitor blocks hippocampal-dependent learning (Shors et al. 2001). Although there is concern about the specificity of chemical inhibitors like the one used in this study, the results provide the first direct evidence that new neurons contribute to the function of adult hippocampus.

\section{Stress Decreases Adult Neurogenesis}

In contrast to the positive effects of exercise and enriched environment, stress significantly down-regulates neurogenesis in adult hippocampus. Gould and McEwen have reported that exposure of marmoset monkeys or tree shrews to intruder stress for only a short period of time $(1 \mathrm{~h})$ down-regulates the rate of neurogenesis in adult hippocampus see Gould et al. (1998). Administration of adrenal-glucocorticoids also decreases neurogenesis in adult hippocampus (Gould et al. 1992; see McEwen1999), indicating that activation of the HPA axis and release of glucocorticoids underlies the downregulation of neurogenesis in response to stress. We have also found that short-term ( $30 \mathrm{~min}$ ) exposure to intermittent footshock decreases neurogenesis by approximately 50\% (Malberg et al. 2000a). More importantly, we have found that exposure to inescapable footshock results in a long-lasting (at least nine days) down-regulation of neurogenesis that is not seen with escapable footshock (Malberg et al. 2000a). The long-lasting down-regulation of neurogenesis resulting from exposure to inescapable footshock also correlates with learned helplessness behavior in this model of depression.

Down-regulation of neurogenesis by exposure to stress, including learned helplessness, raises the possibility that decreased granule cell number could contribute to the reduction in hippocampal volume observed in depressed patients. Decreased neurogenesis could also contribute to selected symptoms of mood disorders, including cognitive abnormalities and loss of inhibitory control of the HPA axis. Additional studies in rodents and postmortem studies in humans will be required to determine if, and to what extent decreased neurogenesis could contribute to the structural changes observed in depression, as well as PTSD. Moreover, the functional relevance of neurogenesis to complex behavioral phenotypes that are observed in models of depression must be determined.

\section{ANTIDEPRESSANT TREATMENT INCREASES ADULT NEUROGENESIS}

In recent years considerable progress has been made in characterizing postreceptor adaptations that occur in response to antidepressant treatment. This has led to the discovery that repeated long-term antidepressant administration up-regulates the CAMP-CREB (cAMP response element binding protein) cascade and expression of brain derived neurotrophic factor (BDNF) in hippocampus (Figure 2) (Nibuya et al. 1995, 1996; Thome et al. 2000). Both CREB and BDNF are known to play a role in synaptic plasticity as well as neuronal survival (see Finkbeiner 2000; Duman et al. 2000).

Based on these findings, studies were initiated to determine if antidepressants influence neuronal remodeling, particularly neurogenesis. The results of this work reveal the exciting discovery that antidepressant treatment increases adult neurogenesis in hippocampus (Figure 3) (Malberg et al. 2000b; Madsen et al. 2000; Manev et al. 2000; see Duman et al. 2000; Jacobs et al. 2000). Up-regulation of neurogenesis is observed with different classes of antidepressants, including 5-HT and NE selective reuptake inhibitors, monoamine oxidase inhibitors, and electroconvulsive seizure. The mood stabilizing agent lithium is also reported to increase neurogenesis in adult hippocampus (Chen et al. 2000). Up-regulation of neurogenesis is dependent on longterm antidepressant administration (two to four weeks), consistent with the time course for the therapeutic action of antidepressant treatment. Other non-antidepressant psychotropic drugs tested, including haloperidol and morphine, do not up-regulate adult neurogenesis. In fact, morphine treatment is reported to decrease neurogenesis in adult hippocampus (Eisch et al. 2000). These results suggest that increased neurogenesis is pharmacologically specific for antidepressants. Further characterization of mature cells resulting from antidepressant administration demonstrates that the cells remain viable four weeks after antidepressant treatment and that approximately $75 \%$ of these cells express markers of neurons (Malberg et al. 2000b).

We have also initiated studies to determine if antidepressant treatment blocks or reverses the down-regulation of neurogenesis resulting from stress. As described above, we have found that exposure to inescapable footshock in the learned helplessness model of depression results in a long lasting down-regulation of neurogenesis that is not observed with escapable footshock. When animals exposed to inescapable shock are treated 


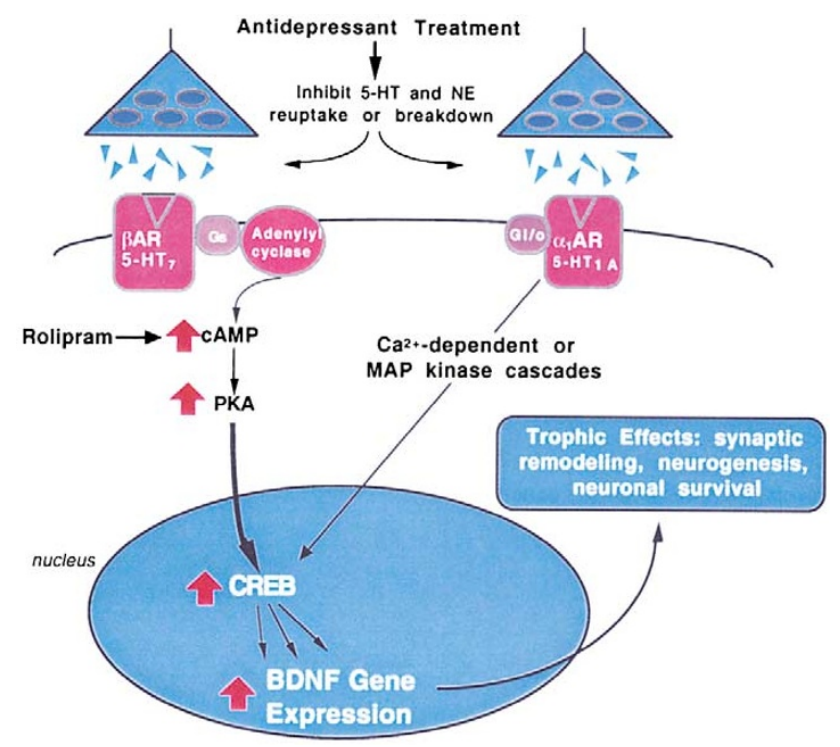

Figure 2. Postreceptor adaptations in response to longterm antidepressant treatment. Antidepressant treatment up-regulates the cAMP cascade at several intracellular sites, including increased coupling of Gs, the stimulatory GTPbinding protein, with adenylyl cyclase, increased levels cAMP-dependent protein kinase (PKA), and increased function and expression of cAMP response element binding protein (CREB) (see Duman et al. 2000). CREB can also be regulated by $\mathrm{Ca}^{2+}$-dependent or microtubule-associated protein (MAP) kinases. One of the target genes regulated by the CAMP-CREB cascade and antidepressant treatment is brain derived neurotrophic factor (BDNF). Up-regulation of CREB and BDNF could contribute to neurotrophic effects of antidepressants, including regulation of adult neurogenesis. with an antidepressant, fluoxetine in this experiment, the behavioral helplessness is reversed. Moreover, we found that fluoxetine treatment also reversed the decrease in neurogenesis (Malberg et al. 2000a).

The results of these studies indicate that up-regulation of neurogenesis would opposes the actions of stress and raise the possibility that increased neurogenesis could contribute to the actions of antidepressant treatment. Additional studies will be needed to directly test this hypothesis. As described above approaches to block adult neurogenesis using either chemical inhibitors or transgenic approaches must be conducted to test the role of neurogenesis in behavioral models of depression and in the action of antidepressant treatment . Analysis of granule cell number in postmortem hippocampus from depressed patients with or without medication at the time of death must also be conducted. These types of studies will contribute to a more complete understanding of the cellular actions of antidepressants and how they relate to depression.

\section{FACTORS INVOLVED IN THE REGULATION OF ADULT NEUROGENESIS}

One of the goals of our work is to understand the molecular mechanisms underlying the actions of antidepressant treatment and we have extended this to regulation of neurogenesis. A brief review of reports on factors involved in the regulation of neurogenesis is provided and then a more detailed description of the work from our laboratory on the role of the cAMPCREB cascade.
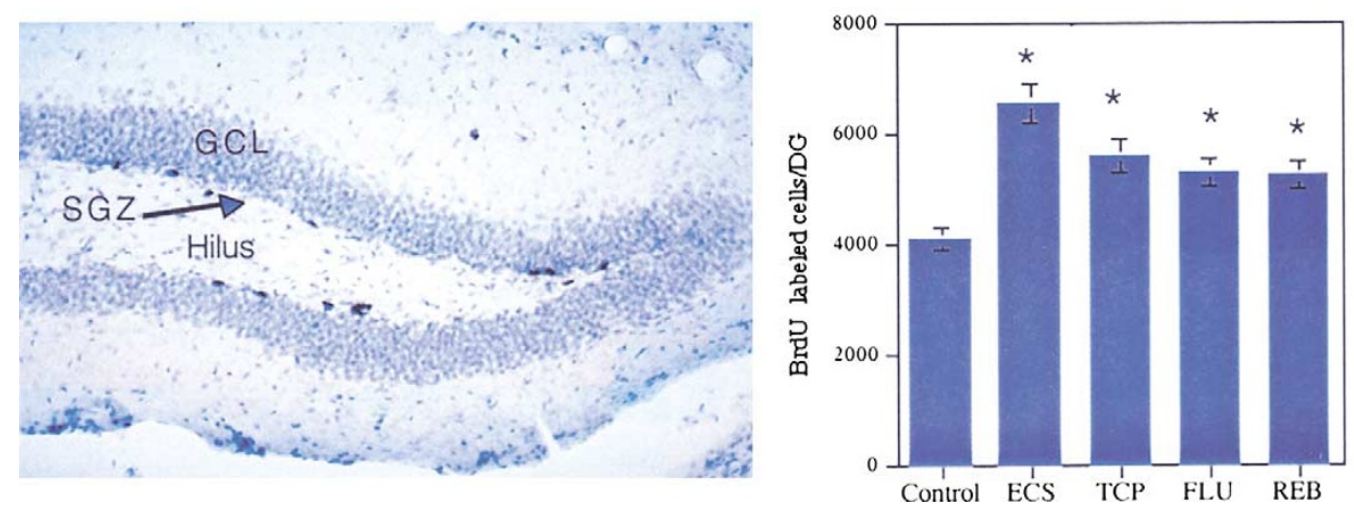

Figure 3. Antidepressant treatment increases neurogenesis in adult hippocampus. The left panel shows a typical section of dentate gyrus that has undergone immunohistochemical analysis for bromodeoxyuridine (BrdU), the thymidine analog used to label newborn cells. The darker cells in the subgranular zone (SGZ) represent BrdU-positive cells. Chronic antidepressant treatment increases the number of BrdU-positive cells, determined $24 \mathrm{~h}$ after BrdU administration. The antidepressants tested include electroconvulsive seizure (ECS), a monoamine oxidase inhibitor tranylcypromine (TCP), a 5-HT selective inhibitor fluoxetine (FLU) and a norepinephrine selective reuptake inhibitor reboxetine (REB). The results are expressed as the total number of BrdU-labeled cells per dentate gyrus and are the mean \pm SEM of eight separate animals. $p<$ .05 compared with control (ANOVA and Tukey's post-hoc test). 


\section{Regulation of Adult Neurogenesis by Neurotrophic Factors}

Previous studies have reported that neurogenesis is regulated by a number of growth factors, including epidermal growth factor, insulin-like growth factor-1, and fibroblast growth factor-2 (Figure 4) (see Cameron et al. 1998). There are also reports that neurotrophic factors, including BDNF, influence neurogenesis. Incubation of cultured progenitor cells with BDNF is reported to increase the differentiation of cells into neurons (Palmer et al. 1997). There is also an in vivo study where BDNF administered intraventricularly was found to increase neurogenesis in the subventricular zone, the region that gives rise to new neurons in the olfactory bulb (Zigova et al. 1998). Unfortunately, the hippocampus was not examined in this study. In preliminary work, we have examined BDNF heterozygous knockout mice to address this question. Homozygous BDNF null mutant mice die shortly after birth so the influence of complete deletion of BDNF could not be determined. In BDNF heterozygous mice there was no difference in the number of newborn cells compared with that in the hippocampus of wild-type littermates. This suggests that half the normal complement of BDNF may be sufficient to support adult neurogenesis. Alternatively, the results may indicate that BDNF is not a critical factor for regulation of neurogene-

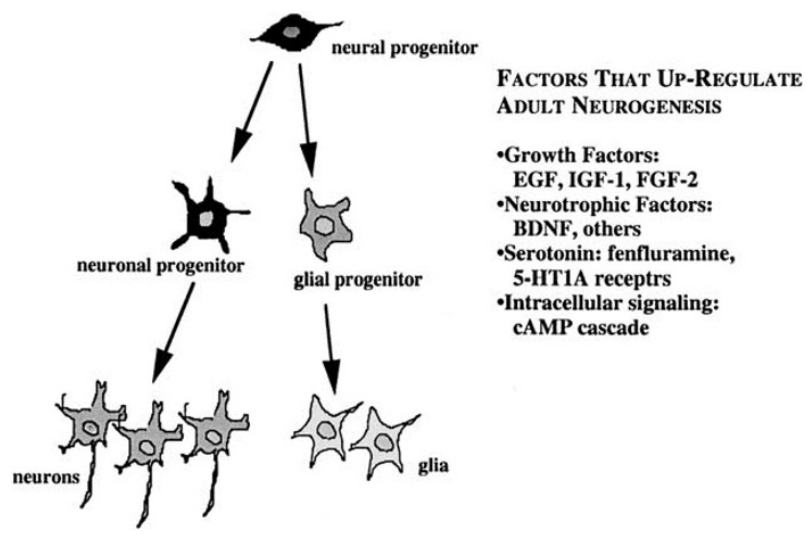

Figure 4. Some factors involved in the regulation of adult neurogenesis. Although much more work remains to identify the factors that control adult neurogenesis, there is evidence that certain growth factors, neurotrophic factors, neurotransmitters, and signal transduction pathways influence adult neurogenesis. A brief discussion of some of these factors is provided in the text. Although the identity of the neural progenitor cells in the subgranular zone of the hippocampus has not been determined studies in the subventricular zone demonstrate that the progenitors are actually astroglial cells that have the potential to divide and differentiate into either neurons or glia (Doetsch et al. 1999). Work is currently being conducted to characterize the hippocampal neural progenitor cells. sis in hippocampus. It will also be interesting to determine if BDNF null mutant mice are more vulnerable to stress, since stress is reported to decrease levels of BDNF, as well as neurogenesis. Heterozygous mice should be useful for these studies because stress decreases levels of BDNF by about 50\% (Smith et al. 1995).

\section{Regulation of Neurogenesis by Serotonin}

There is not much information on neurotransmitter regulation of neurogenesis, although there are studies demonstrating that the 5-HT system can influence the rate of new cell birth in adult hippocampus. Lesion of the 5-HT system is reported to decrease neurogenesis (Brezun and Daszula 2000) and preliminary studies demonstrate that administration of fenfluramine, which causes release of 5-HT, increases adult neurogenesis in hippocampus (Jacobs et al. 1998). In contrast, administration of a $5-\mathrm{HT}_{1 \mathrm{~A}}$ antagonist, WAY 100,635, blocks fenfluramine-induction of neurogenesis, as well as the basal rate of neurogenesis in the absence of fenfluramine (Jacobs et al. 1998). These studies suggest that regulation of the 5-HT system and 5- $\mathrm{HT}_{1 \mathrm{~A}}$ receptors could contribute to the induction of adult neurogenesis by antidepressants, at least the effect of a 5-HT selective reuptake inhibitor. It will be important to directly test the role of $5-\mathrm{HT}_{1 \mathrm{~A}}$, as well as other 5-HT and NE receptor subtypes, in the actions of antidepressant treatments.

\section{Role of the CAMP-CREB Cascade in Adult Neurogenesis}

There is very little information on the intracellular signal transduction pathways that regulate adult neurogenesis. There is one report that activation of the cAMP pathway in cultured progenitor cells increases neuronal differentiation (Palmer et al. 1997). The potential role of the cAMPCREB cascade in neurogenesis is of particular interest because of our previous studies demonstrating that antidepressant treatment up-regulates this pathway (Figure 2) (see Duman et al. 1997, 2000). One piece of evidence of particular interest was discovered when we were studying the influence of antidepressants on CREB phosphorylation. We reported that antidepressant treatment increases the phosphorylation of CREB, measured with a phospho-specific antibody (Figure 5) (Thome et al. 2000). The additional point of interest in these studies was that under basal conditions there was prominent expression of phospho-CREB in cells within, or adjacent to, the subgranular zone where the progenitor cells are located (Figure 4). The finding that phospho-CREB immunoreactivity under basal conditions in the subgranular zone together with our previous work on antidepressant regulation of CREB prompted additional studies to characterize the role of the CAMP-CREB cascade in adult neurogenesis. 

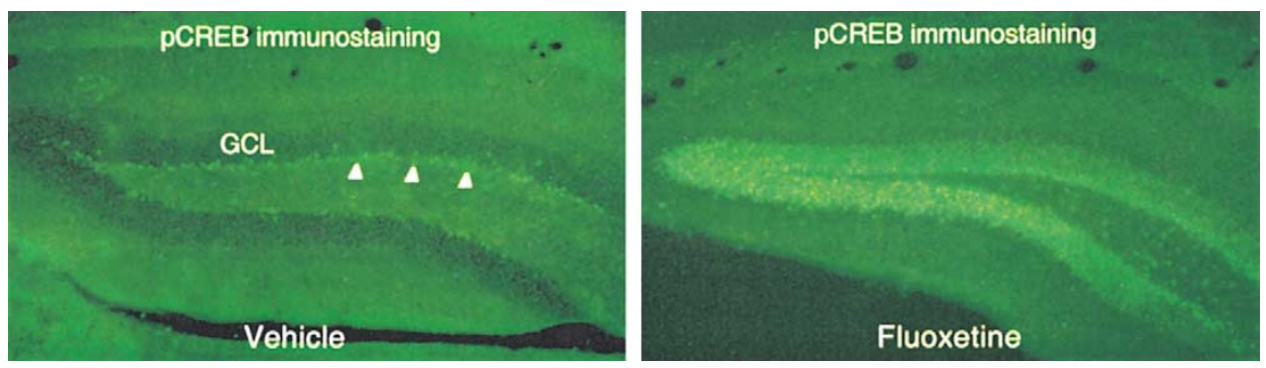

Figure 5. Localization of phospho-CREB (pCREB) in dentate gyrus. Chronic antidepressant treatment increases levels of pCREB, determined by immunohistochemistry with a pCREB specific antibody (see Thome et al. 2000). In vehicle treated controls the expression of pCREB was observed in cells located within or adjacent to the subgranular zone (arrowheads). This suggests that pCREB may influence neurogenesis under basal conditions, as well as in response to antidepressant treatment. It is not known if antidepressant treatment increases the expression of pCREB staining in the subgranular zone above that already present under basal conditions. Higher power examination indicates that pCREB staining is in the nuclei of granule cells (not shown).

In one series of studies a chemical inhibitor of cAMPspecific phosphodiesterase type IV (PDE4), the enzyme responsible for breakdown of CAMP, was used to activate the cAMP pathway. Previous studies demonstrate that a selective PDE4 inhibitor, rolipram, increases the expression of BDNF in hippocampus and this drug was used for the neurogenesis experiments (Nibuya et al. 1996). In preliminary studies we have found that chronic administration of rolipram increases adult neurogenesis in hippocampus (Kim et al. 2000). Up-regulation of neurogenesis by rolipram was dependent on repeated administration (two weeks), similar to the effect of antidepressants. We also found that rolipram increased levels of phospho-CREB in hippocampus, similar to the effect of antidepressant treatment. These findings suggest that activation of the CAMP-CREB cascade regulates specific gene targets that influence neurogenesis.

To more directly test this possibility we have used the tetracycline-inducible transgenic approach to overexpress a dominant negative mutant of CREB in hippocampus (see Chen et al. 1998). The dominant negative CREB contains a mutation of the Ser ${ }^{133}$ site, which is required for phosphorylation-induced transactivation of CREB. Overexpression of mutant CREB in hippocampus significantly decreased neurogenesis in adult hippocampus (Nakagawa et al. 2000). Combined with the rolipram studies, this work demonstrates that activation of the CAMP-CREB cascade up-regulates neurogenesis in hippocampus. The specific CREB-induced gene that leads to induction of neurogenesis is currently being investigated. Studies are also being conducted to determine if the influence of the CAMP-CREB cascade occurs directly on the neural progenitor cells or if the effect is indirect and occurs via expression and release of a factor from surrounding cells. In preliminary studies, we have not been able to detect phospho-CREB in newborn cells that have recently divided. This sug-

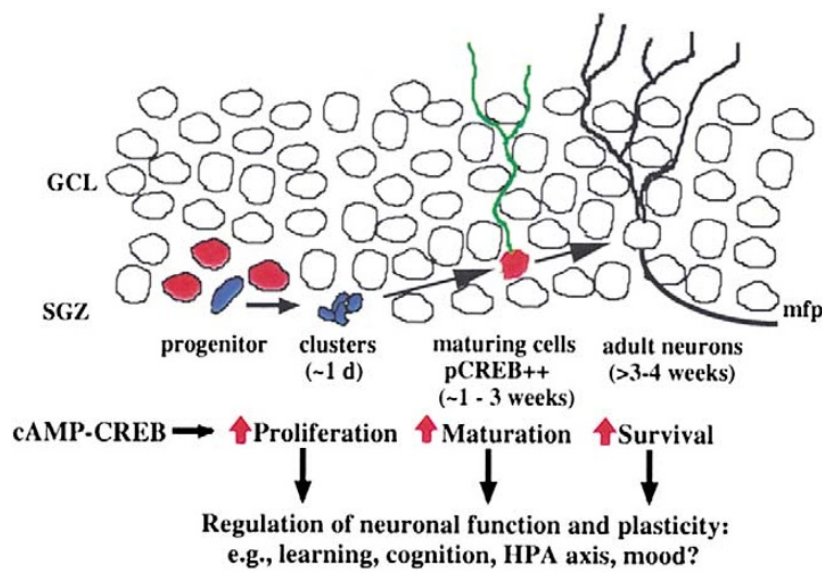

Figure 6. Role of the CAMP-CREB cascade in adult neurogenesis. Preliminary studies indicate that activation of the CAMP-CREB cascade increases neurogenesis in dentate gyrus. Up-regulation of neurogenesis could occur via activation of the cAMP cascade in progenitor cells or in surrounding cells which then release a proliferation factor. The cells colored in red represent phospho-CREB ( $\mathrm{pCREB}$ ) positive cells. In addition, pCREB is colocalized with polysialic acidneural cell adhesion molecular (PSA-NCAM), a marker of neurons that are maturing and/or undergoing morphogenic remodeling. Colocalization of pCREB and PSA-NCAM in BrdU-positive newborn cells is observed only during a critical period of cell maturation (one to three weeks) when the neurogenesis is required for hippocampal-dependent learning (Shors et al. 2001). This suggests that pCREB may play a role in determining the functional fate of maturing neurons in adult hippocampus. It is possible that pCREB is also involved in the regulation of depression-related functions governed by granule cells, including regulation of the HPA axis and even mood. Although hippocampus does not directly influence mood, connections with other brain regions such as the amygdala and prefrontal cortex could result in regulation of emotional states. 
gests that either induction of phospho-CREB is transient or that activation of the CAMP-CREB cascade occurs in the surrounding cells.

\section{Colocalization of Phospho-CREB with Markers of Maturing Neurons}

There are multiple stages at which the process of neurogenesis can be regulated. In addition to proliferation, the maturation, differentiation and survival of newborn cells can be influenced. We have begun to examine the influence of the CAMP-CREB cascade, as well as antidepressant treatment, on these other stages of neurogenesis. One of the ways that maturation of neurons can be studied is to use markers of neurons that are immature or that are undergoing remodeling. One such marker is polysialic acid-neural cell adhesion molecule (PSANCAM). Expression of PSA-NCAM on the cell surface attenuates cell adhesion because of its large hydration volume and negative charge, making it an attractive molecule to mediate changes in cellular remodeling during development and in adult brain (Kiss and Rougon 1997). Although PSA-NCAM disappears from most of the CNS after early postnatal development it continues to be expressed in regions of the brain capable of cellular remodeling and neurogenesis, including the granule cell layer of the hippocampus. PSA-NCAM is reported to play a role in long-term potentiation and other forms of activity dependent cellular remodeling (see Muller et al. 1996; Becker et al. 1996).

In preliminary studies we have examined the interaction of PSA-NCAM and phospho-CREB during the maturation of newborn cells in hippocampus. As reported previously, PSA-NCAM is not expressed in these newborn cells until they begin to extend processes at approximately one week after birth, and then it is continuously expressed up week 3 . Interestingly, we find that the time course for expression of phospho-CREB is exactly the same as that for PSA-NCAM. Tripleimmunohistochemical analysis demonstrates colocalization of PSA-NCAM, phospho-CREB, and bromodeoxyuridine (BrdU) between one and three weeks after cells are born, but not at shorter $(1 \mathrm{~d})$ or longer $(4$ weeks) time points. This suggests that phospho-CREB, like PSA-NCAM, is involved in the maturation and/or differentiation of neurons. We also have preliminary evidence that CREB may influence the expression of PSA-NCAM. The promoter activity of one of the enzymes responsible for the synthesis of PSA-NCAM, a polysialyltransferase, is increased by activation of the CAMP-CREB cascade.

The expression of phospho-CREB, as well as PSANCAM during the 1 to 3-week time frame of neurogenesis, is particularly interesting in light of the recent study from Shors and Gould on neurogenesis and hippocampal-dependent learning (Shors et al. 2001). They found that there was a critical period of neuronal maturation of approximately two weeks that was required for neurogenesis-dependent learning. If neurogenesis was inhibited for a shorter period of time learning was not blocked. They concluded that new neurons must be at a specific stage of maturation to be capable of supporting hippocampal-dependent learning. Previous studies have demonstrated a role for CREB in other forms of cellular and behavioral learning and memory (see Silva et al. 1998). Localization of phospho-CREB during the critical period of granule cell maturation suggests that this transcription factor may play a role in deciding the functional fate of these new neurons (Figure 6). It is possible that new granule cells are also involved in other hippocampal-dependent functions, including regulation of the HPA axis as well as depression related symptoms, and that phospho-CREB contributes to these cellular decisions. Further insight into the functional role of CREB may also be provided by identification of the neurotransmitters and/or neurotrophic factors that control the phosphorylation of CREB during this critical phase of functional maturation.

\section{FUTURE PERSPECTIVES}

Reports from basic and clinical studies demonstrate that in addition to neurochemical alterations, synaptic remodeling and structural alterations contribute to the pathophysiology and treatment of stress-related mood disorders. Although additional studies must be conducted in humans, the discovery of neurogenesis in adult brain and of the environmental and pharmacological factors that regulate neurogenesis represent major advances in the fields of neurobiology and psychiatry. Further progress and breakthroughs in basic neurobiology will continue to provide new avenues of research to characterize the cellular basis of complex behavioral illnesses like depression. The findings discussed in this paper will help shape future studies to identify the cellular alterations that contribute to psychiatric illness, including analysis of more discrete forms of neuronal remodeling (e.g., synapse formation and spine density). Moreover, continued research on the molecular mechanisms that control neurogenesis and neuronal remodeling will result in new strategies for the development of novel therapeutic interventions.

\section{REFERENCES}

Becker C, Artola A, Gerardy-Schahn R, Becker T, Welzl H, Schachner M (1996): The polysialic acid modification of the neural cell adhesion molecule is involved in spatial learning and hippocampal long-term potentiation. J Neurosci Res 45:143-152

Brezun JM, Daszula A (2000): Serotonin may stimulate gran- 
ule cell proliferation in the adult hippocampus, as observed in rats grafted with foetal raphe neurons. Eur J Neurosci 12:391-396

Bremner JD, Randall P, Scott TM, Bronen RA, Seibyl JP, Southwick SM, Delaney RC, McCarthy G, Charney DS, Innis RB (1995): MRI-based measurement of hippocampal volume in patients with combat-related posttraumatic stress disorder. Am J Psychiatry 152:973-981

Bremner J, Narayan M, Anderson ER, Staib LH, Miller H, Charney DS (2000): Smaller hippocampal volume in major depression. American J Psychiatry 157:115-117

Cameron HA, Hazel TG, McKay RD (1998): Regulation of neurogenesis by growth factors and neurotransmitters. J Neurobiol Aug 36(2):287-306

Cameron HA, McKay RDG (2001): Adult neurogenesis produces a large pool of new granule cells in the dentate gyrus. J Comparative Neurology 435:406-417

Chen J, Kelz MB, Zeng G, Sakai N, Steffen C, Shockett PE, Picciotto MR, Duman RS, Nestler EJ (1998): Transgenic animals with inducible, targeted gene expression in brain. Mol Pharmacol 54:495-503

Chen G, Rajkowska G, Du F, Seraji-Bozorgzad N, Manji HK (2000): Enhancement of hippocampal neurogenesis by lithium. J Neurochem 75(4):1729-1734

Doetsch F, Caille I, Lim DA, Garcia-Verdugo JM, AlvarezBuylla A (1999): Subventricular zone astrocytes are neural stem cells in the adult mammalian brain. Cell 97:1-20

Duman RS, Heninger GR, Nestler EJ (1997): A molecular and cellular theory of depression. Arch Gen Psychiatry 54:597-606

Duman RS, Malberg J, Nakagawa S, D'Sa C (2000): Neuronal plasticity and survival in mood disorders. Biol Psychiatry 48:732-739

Eisch AJ, Barrot M, Schad CA, Self DW, Nestler EJ (2000): Opiates inhibit neurogenesis in the adult rat hippocampus. Proc Natl Acad Sci 97:7579-7584

Finkbeiner S (2000): CREB couples neurotrophin signals to survival messages. Neuron 25:11-14

Gage F (2000): Mammalian neural stem cells. Science 287: $1433-1438$

Gould E, Cameron HA, Daniels DC, Wooley CS, McEwen BS (1992): Adrenal hormones suppress cell division in the adult rat dentate gyrus. J Neurosci 12:3642-3650

Gould E, Tanapat P, McEwen BS, Flugge G, Fuchs E (1998): Proliferation of granule cell precursors in the dentate gyrus of adult monkeys is diminished by stress. Proc Natl Acad Sci USA 95:3168-3171

Gould E, Tanapat P, Hastings NB, Shors TJ (1999): Neurogenesis in adulthood: a possible role in learning. Trends Cogn Sci 3:186-192

Gross CG (2000): Neurogenesis in the adult brain: death of a dogma. Neurosci 1:67-73

Jacobs B, Tanapat P, Reeves A, Gould E (1998): Serotonin stimulates the production of new hippocampal granule neurons via the 5HT1A receptor in the adult rat. Los Angeles, Society for Neuroscience Abstracts 24:1992.

Jacobs B, van Praag H, Gage FH (2000): Adult brain neurogenesis and psychiatry: a novel theory of depression. Mol Psychiatry 5:262-269
Kim J-E, Nakagawa S, Duman RS (2000): Chronic administration of rolipram, a cAMP phosphodiesterase-4 inhibitior, upregulates neurogenesis in the adult mouse hippocampus. Soc Neurosci Abstr 26:2316

Kiss J, Rougon G (1997): Cell biology of polysialic acid. Curr Opin Neurobiol 7:640-646

Madsen TM, Treschow A, Bengzon J, Bolwig TG, Lindvall O, Tingström A. (2000): Increased neurogenesis in a model of electroconvulsive therapy. Biol Psychiatry 47: 1043-1049

Malberg JE, Eisch AJ, Nestler EJ, Duman RS (2000a): Effects of antidepressant treatment and learned helplessness training on hippocampal neurogenesis in adult rat. Soc Neurosci Abstr 26:1044

Malberg JE, Eisch AJ, Nestler EJ, Duman RS (2000b): Chronic antidepressant treatment increases neurogenesis in adult hippocampus. J Neurosci 20:9104-9110

Manev H, Uz T, Smalheiser NR, Manev R (2000): Antidepressants alter cell proliferation in the adult brain in vivo and in neural cultures in vitro. Eur J Pharmacol 411:67-70

McEwen BS (1999): Stress and hippocampal plasticity. Annu Rev Neurosci 22:105-122

Muller D, Wang C, Skibo G, Toni N, Cremer H, Calaora V, Rougon G, Kiss JZ (1996): PSA-NCAM is required for activity induced synaptic plasticity. Neuron 17:413-422

Nakagawa S, Kim J-E, Lee R, Chen J, Duman RS (2000): CREB plays a critical role in the survival of newborn cells in the adult hippocampus. Soc Neurosci Abstr 26:2317.

Nibuya M, Morinobu S, Duman RS (1995): Regulation of BDNF and trkB mRNA in rat brain by chronic electroconvulsive seizure and antidepressant drug treatments. J Neurosci 15:7539-7547

Nibuya M, Nestler EJ, Duman RS (1996): Chronic antidepressant administration increases the expression of cAMP response element binding protein (CREB) in rat hippocampus. J Neurosci 16:2365-2372

Palmer TD, Takahashi J, Gage FH (1997): The adult rat hippocampus contains primordial neural stem cells. Mol Cell Neurosci 8:389-404

Sheline YI, Wany P, Gado MH, Csernansky JG, Vannier MW (1996): Hippocampal atrophy in recurrent major depression. Proc Natl Acad Sci USA 93:3908-3913

Sheline YI, Sanghavi M, Mintun MA, Gado MH (1999): Depression duration but not age predicts hippocampal volume loss in medically healthy wormen with recurrent major depression. J Neurosci 19(12):5034-5043

Shors T, Miesegaes G, Beylin A, Zhao M, Rydel T, Gould E (2001): Neurogenesis in the adult is involved in the formation of trace memories. Nature 410:372-376

Silva AJ, Kogan JH, Franklind PW, Kida S (1998): CREB and memory. Ann Rev Neurosci 21:127-148

Smith MA, Makino S, Kvetnansky R, Post RM (1995): Stress alters the expression of brain-derived neurotrophic factor and neurotrophin-3 mRNAs in the hippocampus. J Neurosci 15:1768-1777

Steffens D, Byrum CE, McQuoid DR, Greenberg DL, Payne ME, Blichington TF, MacFall JR, Krishnan KRR (2000): Hippocampal volume in geriatric depression. Biol Psychiatry 48:301-309 
Tanapat P, Hastings NB, Reeves AJ, Gould E (1999): Estrogen stimulates a transient increase in the number of new neurons in the dentate gyrus of the adult female rat. J Neurosci 19:5792-5801.

Thome J, Sakai N, Shin K, Steffen C, Zhang YJ, Impey S, Storm D, Duman RS (2000): cAMP response elementmediated gene transcription is upregulated by chronic antidepressant treatment. J Neurosci 20:4030-4036 van Praag H, Kempermann J, Gage FH (2000): Neural consequences of environmental enrichment. Nature Reviews / Neurosci 1:191-198.

Zigova T, Pencea V, Wiegand S, Luskin M (1998): Intraventricular administration of BDNF increases the number of newly generated neurons in the adult olfactory bulb. Mol Cell Neurosci 11:234-245 